\title{
True Motion in Relative Displays
}

\author{
from A. L. P. Milwright \\ (Admiralty Surface Weapons Establishment)
}

THE system proposed by Manley $(15,172)$, to produce true motion on relative displays seems to suffer from two serious disadvantages which were not referred to. The first is that there would be a serious degradation in the resolution of the stored picture, since every time an original echo is rewritten into one of the Tenicon storage tubes the echo size would be increased both in range and bearing by the size of the beam spot. No doubt the full effect of this increase could be reduced by the introduction of pulse shaping circuits between the read-out and write-in points, but nevertheless the original small echo would be considerably increased in size if a number of read-out write-in operations were carried out as would be the case if the information was stored for a sufficiently long time to show true track. As an example, without applying any special pulse shaping techniques a small echo, two spot diameters long and one spot diameter wide, would be increased in size by 65 spot diameters if stored for 30 seconds (i.e. Io scans of the aerial) and would be increased by 1890 spot diameters if stored for 3 minutes.

The second disadvantage is probably the more serious. Since any signal entering the system remains there until the stored information is cancelled, the effect of interference would be to flood the screen completely in a short space of time. In the case of receiver noise and sea clutter the effect could be reduced by the introduction of signal clipping and swept gain but at the expense of receiver sensitivity. However, it would be extremely difficult to reduce the effect of interference from another ship's radar on the same frequency, and interference of this type would be received from ships within at least an 8 miles radius irrespective of the relative position of the two radar aerials. In congested waters such as the Dover Strait there is a high probability of receiving this type of interference from a number of ships, but with the proposed system the stored picture would be completely saturated by the interference from only one ship in a very short time.

\section{Mr. B. W. Manley comments:}

If an echo of size $A_{0}$ is stored on the Tenicon target, and its size is increased by an amount $(p \times a)$ at each transference, where $a$ is the spot size and $p$ is a numerical factor, then after $n$ such transferences the size $A_{n}$ of the stored signal is given by:

$$
A_{n}=A_{0}+n p a
$$

Milwright seems to have arrived at a form :

$$
A_{n}=\sum_{k=0}^{k=n}\left(A_{0}+k p a\right)
$$

which would indicate an alarming and unobserved rate of growth. What small amount of evidence we have at present on the use of the Tenicon in a 'ping-pong' mode, indicates that $p$ is less than unity. After three minutes scanning, therefore, 
the elemental echo would certainly be less than 60 spot diameters, rather than the 1890 which Milwright suggests.

The use of pulse shaping would, of course, improve matters considerably in this respect, but would possibly introduce the second problem to which Milwright refers. The signal from one storage tube could be processed so that synthetic pulses of fixed length and amplitude, produced in response to signals above a selected amplitude, would be applied to the input of the second storage tube. In these circumstances, any such signal, once stored, would be retained until it passed off the target by virtue of the motion of the observing ship. Interference might thus be stored and could confuse the picture. If the original signal were used to build up the true track, then by setting the loop gain of the storage cycle to be less than unity, a trace which would diminish in intensity with time would be observed. By varying the loop gain, the trace persistence could be adjusted so that interference would be prevented from building up excessively.

As I have already said in my conclusion to the article, such a system may add confusion rather than clarity to a complex situation, but this can best be determined by experiment. The method would at least have the advantage of being additive to the normal relative display. In a situation where it is not contributing useful information it could be switched out to leave the relative display.

\title{
Sundials for Navigators
}

\author{
from Captain Mario Bini \\ (Italian Navy)
}

I. Having some time ago been requested to design a sundial for the Hydrographic Institute of Genoa (Fig. I) I went through various books and found that the methods indicated for drawing sundials were all based on graphical constructions. Although quite interesting these methods have the inconvenience of all graphical solutions, that they require constructions which, besides being complicated, turn out to be rather inaccurate, especially when lines cross at small angles, as inevitably happens outside a limited central area of the drawing. To avoid this I worked out a numerical system, related to the familiar methods of astro-navigation, which, by means of simple formulae, gives the coordinates of the points necessary to design a sundial. As the bibliography I examined is, of course, limited, I am not in a position to state whether this method of calculation is new or not; I describe it here hoping it may interest people who have sometimes wondered how sundials are made.

2. Sundials are based on the principle that a gnomon stylus casts its shadow, over a datum plane, along a line having a direction depending only on time, provided the stylus is set parallel to the Earth's axis.

The stylus OS (Fig. 2) is contained in the plane of all hour circles, its shadow corresponding to different positions of the Sun (such as I, 2, 3) along the same 